\title{
A Framework to Support Interoperability among Semantic Resources
}

\author{
C. Lima ${ }^{1}$, C. Ferreira-da-Silva ${ }^{1}$, C. Le Duc ${ }^{1}$, A. Zarli ${ }^{1}$ \\ ${ }^{1}$ CSTB, Centre Scientifique et Technique du Batiment, Routes de Lucioles BP209, \\ 06904 Sophia Antipolis, France \\ \{c.lima, ferreira-da-silva, chanleduc,alain.zarli\}@cstb.fr
}

\begin{abstract}
This paper presents the framework to support the interoperability among Semantic Resources ${ }^{1}$ (SRs) proposed by the FUNSIEC project. FUNSIEC aims at studying the feasibility of building and maintaining an Open Semantic Infrastructure for the European Construction Sector (OSIECS). FUNSIEC adopted a layered approach where the SRs are mapped at the metaschema and schema levels. This process produces the respective OSIECS metaschema and schema, which support the design of the OSIECS kernel - the heart of the OSIECS infrastructure. This paper presents and discusses the elements involved in FUNSIEC's work (e.g. framework, architecture, methodology). Some conclusions and future work complete the paper.
\end{abstract}

\section{Introduction}

The FUNSIEC project touches a dynamic, constantly evolving subject: the meaning of things or in a word, their semantics. Manufacturing industry has faced a worthwhile challenge in the last two decades, finding ways to communicate precisely (i.e. without ambiguity) and indeed good technical results have been achieved (e.g. STEPrelated works, EDI, and more recently the ebXML initiative). The construction industry faced the same challenge and found similar tools to cope, aided by the development and promotion of the Industry Foundation Classes (IFC model \& tools) established by the IAI collaborative initiative [13]. Other projects and initiatives have worked in the same domain and produced related contributions to raise awareness in the whole sector, such as the LexiCon from Stabu [14], the e-COGNOS IST project [5], the CEN/ISSS eConstruction Workshop [14], to name just a few.

FUNSIEC is a continuation of some of these efforts aiming at to evaluate the feasibility of building and maintaining an Open Semantic Infrastructure for the European

1 Semantic Resource is an expression coined in the SPICE project which refers all ontologysimilar entities, such as taxonomies, dictionaries, thesauri, etc.. Two arguments supported this choice: there is no consensus about what ontology really is and there is a myriad of expressions currently available to define ontologies and similar resources. 
Construction Sector (OSIECS), intended mainly to support the e-business needs of Construction. FUNSIEC targets the current situation where standards (both official and de facto), classification systems, taxonomies, ontologies, and related technologies and tools are used in a complementary or even in a completely isolated way to produce the "desirable but feasible" framework where the previously described elements can be combined in an inter-related and holistic fashion.

OSIECS has been created with the SRs currently available that support e-business practices. Following the recommendation provided by the CEN/ISSS eConstruction Workshop [14] the SRs were selected and the most relevant have been mapped among themselves at meta-schema and schema levels respectively. This mapping is expected to produce OSIECS meta-schema and schema.

This paper focuses on the development of OSIECS, describing the FUNSIEC approach to achieving the goal. The document is structured as follows: section 2 describes the context in which the work is carried out; section 3 presents the FUNSIEC approach to develop OSIECS; section 4 discusses the architecture of the OSIECS kernel; section 5 concludes the paper and points out what is next in FUNSIEC.

\section{Context of Work}

As previously mentioned, the FUNSIEC approach to developing OSIECS is based on the selection and mapping of the most relevant SRs. The mapping is made at two levels in a semi-automatic way, with experts and supporting software tools creating the liaisons among the SRs that form the essence of OSIECS. This is, indeed, a kind of pivot providing the necessary bridges to handle semantic queries concerning all the SRs mapped through OSIECS.

\subsection{The FUNSIEC Project}

The FUNSIEC project focuses on the interoperability of semantic resources through the design of the OSIECS infrastructure. FUNSIEC is not a classical R\&D project in the sense that it targets mainly the feasibility of building and maintaining OSIECS at a technical, organisational and business level. As such, the answer must be pragmatic and take advantage of the resources currently available, including public results produced by international initiatives and EC-funded projects. The feasibility study also evaluates the alternatives to maintain semantic mappings amongst the available SRs in order to foster complementarities amongst them and favour the emergence of new electronic services (especially those supporting multiple languages). The development of OSIECS is expected be an effective mechanism to provide answers to the business needs related to the use of SRs.

The main elements involved in the work of FUNSIEC are e-business, standards, and semantic resources (the inputs) and the OSIECS infrastructure and the education dimension (the major outputs). All are evidenced in the FUNSIEC Semantic Experience Centre (FSEC) a kind of hands-on learning experience. The semantic resources are evaluated and mapped among themselves. Standards and recommendations pro- 
vided by standardisation bodies are included in a thorough way in FUNSIEC. The FSEC is, of course, the key element supporting the education axis in FUNSIEC. The (e)business side of the story is related to the very essence of the feasibility study on the use of SRs. How they can be used effectively and how they can provide business advantage are the questions FUNSIEC seeks to answer.

FUNSIEC intends to be an instrument that promotes e-business practices to the construction community. It aims to become a useful and extensible aid relating to the semantic resources theme, a place where people can obtain educational support. Such support will be provided by the creation of a showroom of SRs available and potentially usable by the construction sector in Europe, including usage scenarios, on-line tutorials and demonstrations. FUNSIEC is not about reinventing the wheel; rather it is oriented towards reuse and adoption/adaptation of what seems best in helping the construction sector to be educated in the meaning, development and use of SRs.

\subsection{The FUNSIEC Framework}

The FUNSIEC framework contains three domains, namely User, Application, and Resource (figure 1). The User domain contains the actors involved in the e-business arena. The Application domain holds the e-business areas of application. Finally, the Resource domain offers the SRs used by the application areas and brings in two new elements, namely OSIECS meta-schema and schema. Together these provide the cornerstone supporting the mapping (at different levels) between SRs.

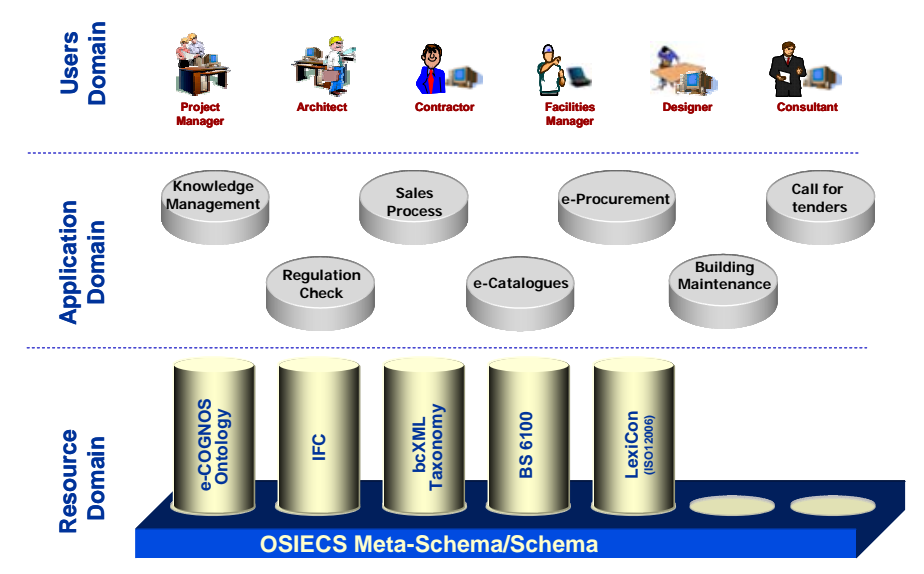

Fig. 1. The FUNSIEC framework

The approach to conceiving OSIECS started with the selection of the SRs that form it. Since they are not described in a uniform language, their respective metaschemas were converted to a single format adopted by FUNSIEC (i.e. the Ontology Web Language - OWL) allowing (semi)automatic detection and validation of commonalities amongst the meta-schemas. This process produces the OSIECS meta- 
schema that supports the mapping amongst the SRs in OSIECS. The OSIECS metaschema is then used to guide the mapping of the entities (i.e., concepts, relations, properties, etc.) defined in each schema of the OSIECS components. The final end result is the OSIECS schema.

\section{The FUNSIEC Approach}

Beyond the "simple" pooling of linguistic resources, FUNSIEC aims at creating a harmonised environment where each resource is clearly characterised (in terms of content, scope, usage, etc.) and situated in the overall map of resources, where semantic links are created among them. Currently, the lack of visibility together with the absence of agreed standards on a large scale are two of the main factors explaining why end-users and service providers are not using exploiting resources more intensively. Moreover, the creation of consistent linguistic resources is most often a very tedious task for content providers with no guarantee of return for the effort. Certainly a major challenge for FUNSIEC is to overcome these problems and create "critical mass" interest and confidence needed to encourage content and service providers. Therefore, the potential risks of failure, along with the success factors, have been carefully evaluated during this feasibility project.

The FUNSIEC approach to developing the OSIECS infrastructure consists of the following steps: (i) identification and selection of the SRs to provide the input for the creation of the OSIECS meta-schema; (ii) analysis of structure, syntax, and semantics of the respective meta-schema of the SRs selected in step $i$; (iii) design of the OSIECS Kernel, a software tool to help creating the OSIECS meta-schema/schema; (iv) semi-automatic conversion of the meta-schemas for the same format; ( $v$ ) semiautomatic mapping of the converted meta-schemas producing the (unified) OSIECS meta-schema; (vi) production of the OSIECS schema from the meta-schema; and (vii) design the OSIECS infrastructure and putting in place a demonstration scenario. These steps are described in more detail in the next sections.

\subsection{The Three Layers Vision in FUNSIEC}

All semantic resources follow some underlying meta-schema and schema, even if not always stated explicitly. In order to enable interoperability among different Semantic Resources it is essential to understand beforehand the meta-schemas they follow. By way of illustration only, figures 2, 3 and 4 show the beXML meta-schema, part of bcBuildingDefinitions taxonomy and a catalogue (bcXML compliant) of products.

The CEN/ISSS eConstruction Workshop ${ }^{2}$ recommends the use of frameworks structured in two levels, namely meta-schema and schema. FUNSIEC considers a third level in this framework, the instances. At the highest level, there are metaschemas that describe very general and abstract concepts that are necessary when

\footnotetext{
2 The CEN/ISSS Workshop on eConstruction was a mechanism to facilitate "e-volution" in European Construction by providing an open discussion forum to achieve the required consensus on a number of interrelated specifications needed for outworking of eConstruction.
} 
structuring an information model and those that describe broadly sharable ideas usually being specific to building construction. From a top-down perspective, specialisation is the axis transforming a meta-schema into instances and generalisation supports the other way round. The lower levels are more detailed and real things whilst higher levels are more abstract and intangible things.

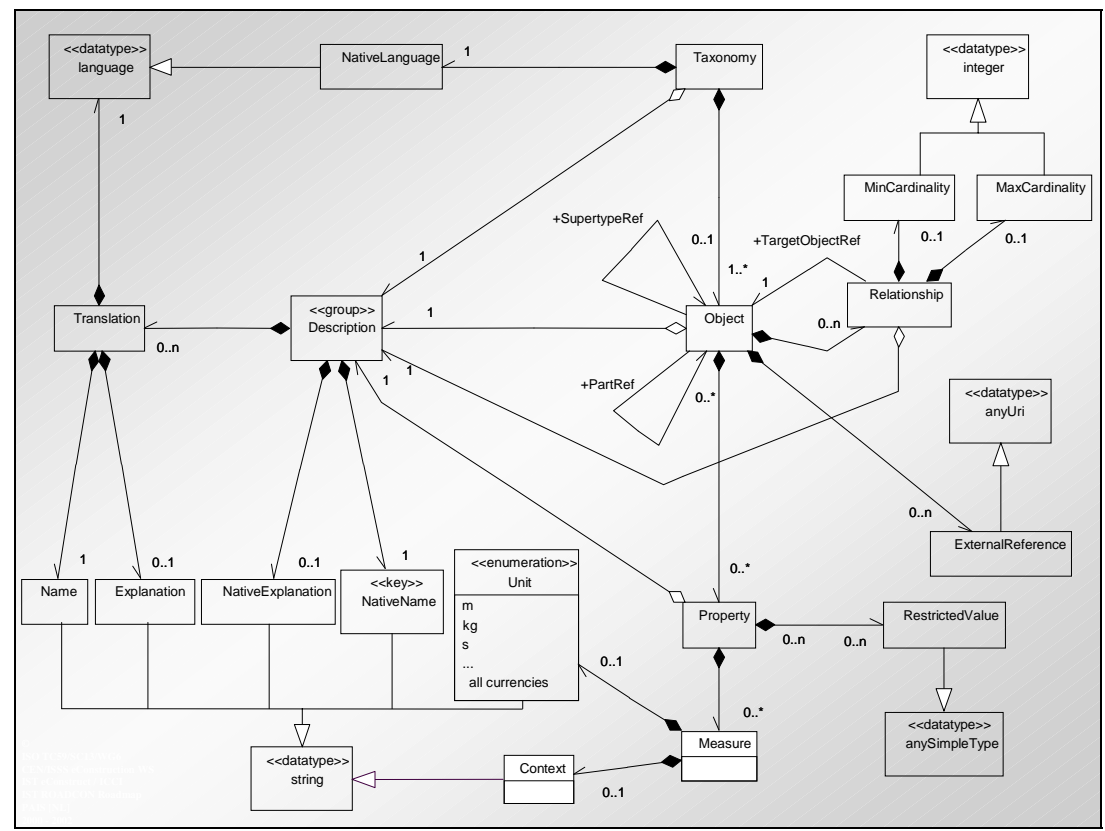

Fig. 2. The bcXML Meta-schema

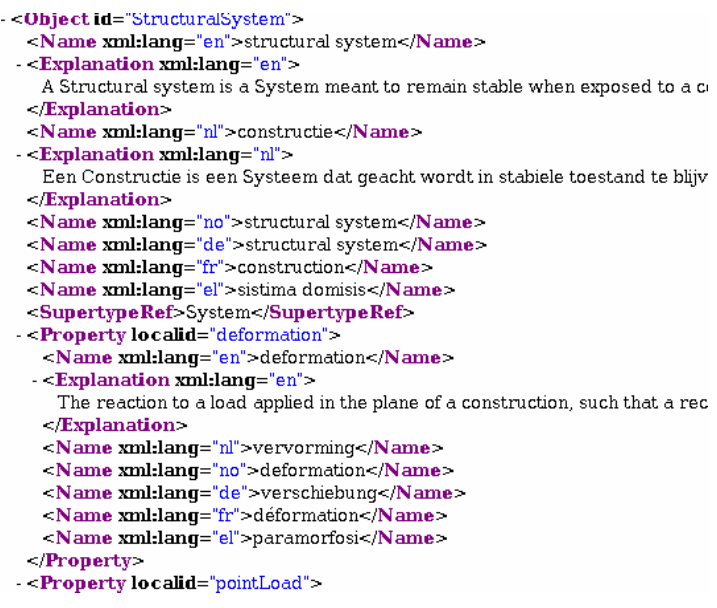

Fig. 3. Partial view of bcBuildingDefinitions taxonomy 
A meta-schema can therefore be regarded as a schema on a high level of abstraction. In the second level, the schema represents an agreed structure expressed in some suitable (mostly formal) language able to describe things on different 'description levels' (e.g. a taxonomy or an ontology). It should be able to handle both definitions and specifications (types and occurrences) of construction-related products and services. Finally, at the bottom level, instances are very specific representations of a schema; for instance a catalogue of products where all the properties that define a given product have the correct values defined (e.g. catalogues of products).

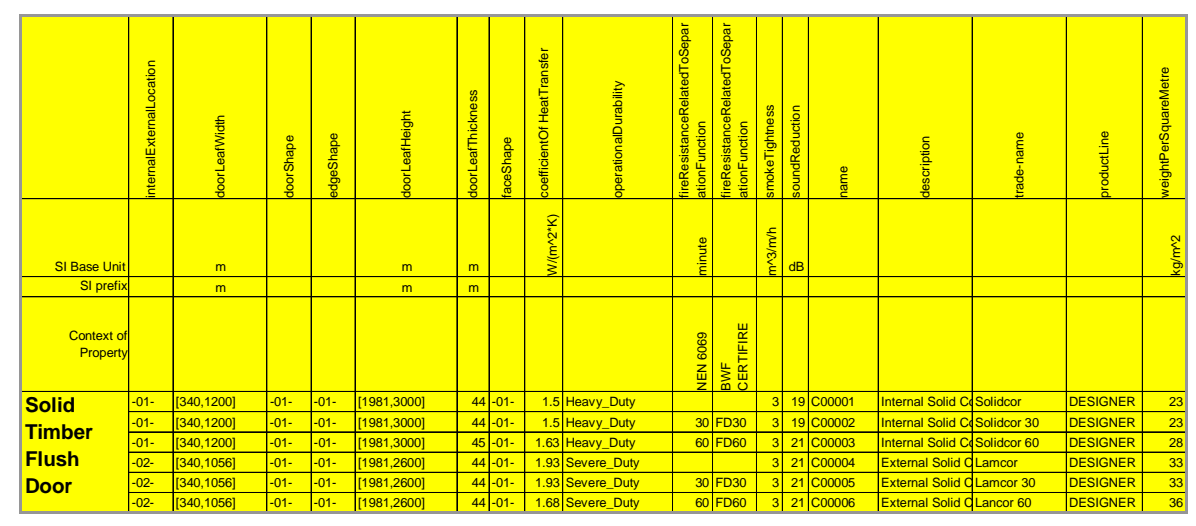

Fig. 4. Typical Door Catalogue Content

\subsection{Mapping the Semantic Resources}

Mapping semantic entities, even if they are formally defined, is a considerable challenge involving several aspects. Quite often it requires identifying pair-wise similarity between entities and computing the best match for them [2]. There are many different possible ways to compute such similarity with various methods designed in the context of data analysis, machine learning, language engineering, statistics, or knowledge representation. FUNSIEC relies on semantic methods [3] to deal with the problem. The basic assumption behind semantic methods is that they aim at discovering relations between (pairs of) entities belonging to different schemata based on the meaning of the two entities (their semantics!).

OSIECS is formed by SRs from the following: bcBuildingDefinitions taxonomy, eCOGNOS, ISO 12006, and IFC. The first step in the development of OSIECS is mapping their meta-schemas. The bcBuildingDefinitions is the taxonomy developed by the eConstruct project in order to demonstrate the power of bcXML ${ }^{3}$, an XMLbased language tailored to the representation of products/services in the construction sector. The bcXML meta-schema is the model of bcXML taxonomy [4].

${ }^{3}$ bcXML primarily supports simple eCommerce communication of products (materials, components, equipment, documents) and services within or across borders. 
The e-COGNOS ontology [5] focuses on construction concepts related to the consistent knowledge representation of (construction) knowledge items. The e-COGNOS ontology comprises two taxonomies (concepts and relations). Those concepts and relations are grounded in the IFC entities, which form the highest level taxonomies.

The primary target of the IFC model is interoperability of software applications in the building and construction sector. IFC classes are therefore defined according to the scope and the abstraction level of software systems dealing with Construction specific content. The entities of the IFC model are grouped in layers where the kernel and core extension layers deal with general, abstract concepts whilst the shared elements and domain layers deal with specialised concepts of the real world [6].

Table 1. Similarities among entities of several meta-schemas

\begin{tabular}{|l|l|l|l|l|}
\hline \multicolumn{1}{|c|}{ Meta-schema } & eCognos & bcXML & ISO 12006-3 & IFC \\
\hline $\begin{array}{l}\text { Item } \\
\text { Semantic Resource } \\
\text { dentification }\end{array}$ & Object (abstract) & Taxonomy & XtdRoot (ABS) & IfcRoot (ABS) \\
\hline Concept & eCognosConcept & Object & XtdObject (ABS) & IfcObject (ABS) \\
\hline Relation & Relation & Relationship & xtdRelationship & IfcRelationship \\
\hline Property & Attribute & Property & xtdProperty & IfcProperty \\
\hline $\begin{array}{l}\text { Assign properties } \\
\text { to objects }\end{array}$ & Attribute & Property & $\begin{array}{l}\text { xtdRelAssign- } \\
\text { sProperties }\end{array}$ & $\begin{array}{l}\text { IfcRelDefined- } \\
\text { ByProperties }\end{array}$ \\
\hline Description & $\begin{array}{l}\text { ObjectCon- } \\
\text { ceptDefinition }\end{array}$ & Description & xtdDescription & $\begin{array}{l}\text { Description (at- } \\
\text { tribute) }\end{array}$ \\
\hline $\begin{array}{l}\text { Reference to an } \\
\text { external resource / } \\
\text { entity }\end{array}$ & - & $\begin{array}{l}\text { Exter- } \\
\text { naReference }\end{array}$ & xtdReference & $\begin{array}{l}\text { IfcExternalRefer- } \\
\text { ence }\end{array}$ \\
\hline
\end{tabular}

The ISO 12006-3 [7] is a Construction specific standard that defines a schema for a taxonomy model, which enables concepts to be defined by means of properties, the grouping of concepts and defining relationships between concepts. Objects, collections and relationships are the basic entities of the model. The role that an object should play can be designated through the model and this provides the capability to define the context in which the object is used [8].

Despite of the differences of the SRs described above found among the metaschemas, the preliminary results produced by analysis in FUNSIEC brought positive signs for the possibility of building a pivot meta-schema bridging (semantically) those SRs. The table 1 presents the partial results of such analysis.

\section{The Architecture of the OSIECS Kernel}

Based on the framework described above, the preliminary version of the OSIECS Kernel is depicted in figure 5. Essentially it covers both meta-schema and schema levels. In the former, the Kernel is formed by: the Syntax Converter, the Semantic Analyser, the Converter, the Detector of Similarities, and the Validator. In the latter, the Kernel relies on the Entities Matcher. 


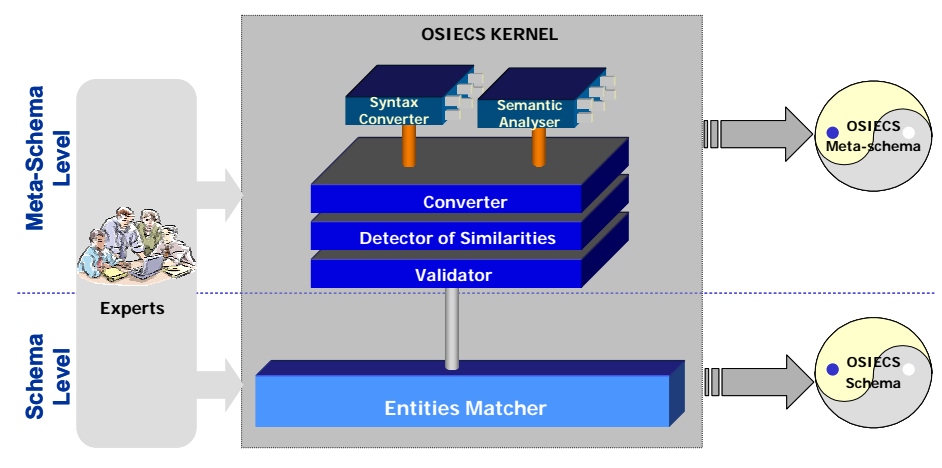

Fig. 5. The OSIECS Kernel

The formalism adopted to represent both OSIECS meta-schema and schema is the OWL language, for two main reasons: $i$ ) the expressiveness; and ii) the explicitness of its semantic representation which allows some automated deduction. Last, but not least, OWL is the ontology representation promoted and recommended by the Semantic Web group.

The Syntax converter and the Semantic analyser work together using the metaschemas of the respective SRs as input in order to produce the conversion rules to be used by the Converter for guiding the production of the OWL meta-schemas for each of the SRs in the Kernel. The Detector of Similarities (which is, indeed, the ONDIL system briefly introduced in section 4.1) works with the OWL-converted metaschemas in creating the OSIECS meta-schema. This meta-schema is then analysed and assessed by the Validator. Then moving one level down, the schemas of the OSIECS components are matched by the Entities Matcher. The output of this process is the OSIECS schema.

With the help of software tools, the experts play a very important role in this process. They act in both levels taking care of: (i) the manual analysis of the SRs and their respective meta-schemas/schemas; (ii) analysis of the rules of conversion; (iii) the assessment of the detection of similarities; (iv) checking of the validation process; and finally $(v)$ the assessment of the output of the Entities Matcher.

\subsection{The ONDIL System}

As previously refereed, within the OSIECS Kernel the ONDIL system is responsible for the detection of similarities among meta-schemas. Broadly speaking, ONDIL provides inference services for Description Logic-based ontologies [9]. The expressiveness of such ontologies allows semantics of modelling languages to be formalised (e.g UML [11], EXPRESS [12]) and to make the semantics as explicit as possible. It is worth emphasising that formal and explicit semantics are crucial to automated deduction.

The ONDIL system comprises three modules, namely ontology management, mediator, and inference engine. The heart of ONDIL is an inference engine that uses the 
structural algorithms developed in [9] for non-standard inferences and the optimised algorithms for standard inferences. ONDIL uses the inference engine to deduce new knowledge using the ontologies (one or many) as the primary source of knowledge. The knowledge deduced is essentially new relations among the ontological concepts. ONDIL considers initially that the ontology manager may need some help to exploit all the possible relationships among the concepts in a single ontology. This help is even more essential when considering several semantic resources (likely) developed independently from each other. The relationships among them (if they exist) are usually only implicit. These relationships can be viewed more as knowledge to be detected rather than knowledge to be predefined in the semantic resources. Therefore, in FUNSIEC the ONDIL system is used to establish mappings among the semantic resources.

\subsection{Syntax Conversion and Semantic Analysis}

As previously explained, the mapping process involves three main elements of SRs, namely the structure, the syntax, and the semantics. The solution most recommended for syntax and semantics problems is to represent (through a conversion) the original SRs in a neutral formal format. These converted versions are then free of syntactical problems. Both structural and semantic-related problems are solved through a semiautomatic process where the experts are aided by software tools. This is where the Converter component (figure 6) comes in. Needless to say, the knowledge of experts is vital to ensure good results.

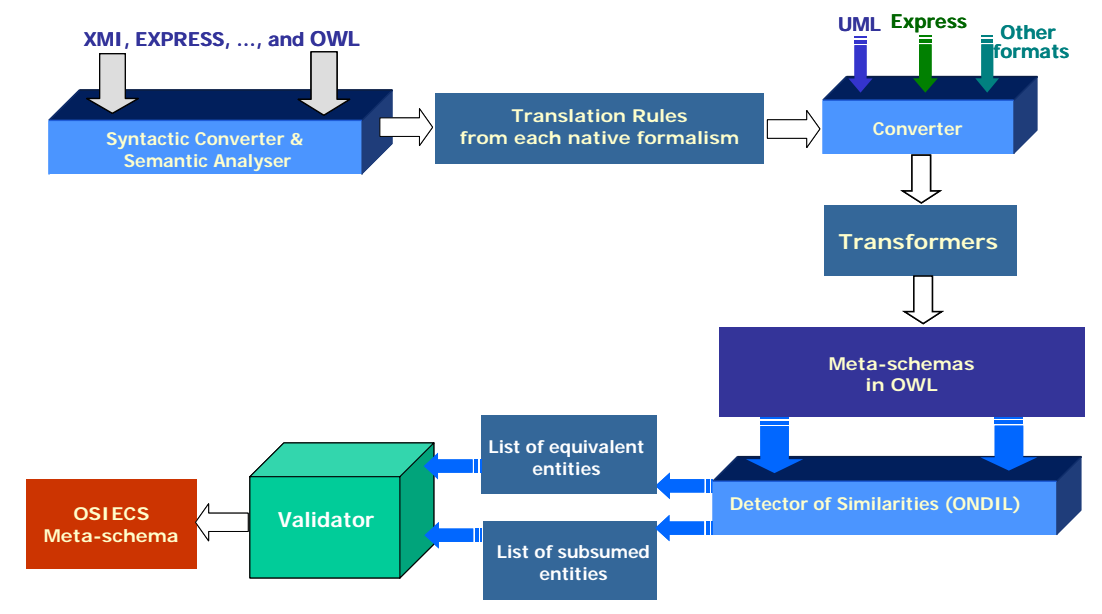

Fig. 6. Creating the OSIECS Meta-schema

The meta-schemas forming OSIECS are originally represented in different formalisms: EXPRESS in ISO 12006-3 and IFC, and UML in e-COGNOS and bcXML.

The Converter works with the meta-schemas in their original formalisms and produces the correspondent OWL versions (figure 6). The experts play a very strategic 
role in this phase. They analyse the SRs meta-schemas and create a set of conversion rules (written in Java) that allows converting each entity from their original format (in a given meta-schema) in OWL. This transformation must preserve the semantics of the entities. The set of rules is used by the JavaCC [10] tool to generate a compiler capable of translating any meta-schema written in the original formalism automatically into OWL. As part of the OSIECS development, two compilers were generated to support the translations of both EXPRESS and UML to OWL. Now the inclusion of new SRs represented in EXPRESS or UML no longer require human intervention.

Table 2 shows the translation of an ENTITY ISO-12006 (in EXPRESS) into OWL. The EXPRESS ENTITY is firstly represented in description logic and this representation is translated into OWL. The class XtdRelAssociates defines a set of instances that satisfy the following property: each instance of class XtdRelAssociates is associated with an instance $I 0$ of class XtdObject via the attribute RelatingObject and with $n$ instances $I 1, \ldots$. In of class XtdObject via the attribute RelatedObjects. The constraint WR1 states that the instance $I 0$ is different from all instances $I 1, \ldots, I n$.

Table 2. An example showing the conversion EXPRESS-OWL

\begin{tabular}{|c|c|c|}
\hline EXPRESS & Description Logic & OWL \\
\hline $\begin{array}{l}\text { ENTITY XtdRelAssociates; } \\
\text { RelatingObject: XtdObject } \\
\text { RelatedObjects: } \\
\text { SET[1 :?] OF XtdObject; } \\
\text { WHERE } \\
\text { WR1: } \\
\text { SIZEOF(QUERY( } \\
\text { Result<*RelatedObjects | } \\
\text { RelatingOb- } \\
\text { ject:=:Result))=0; } \\
\text { END_ENTITY; }\end{array}$ & $\begin{array}{l}\text { relatingObject } \sqsubseteq \\
\text { relationship } \\
\text { relatedObjects } \sqsubseteq \\
\text { relationship } \\
\text { XtdRelAssociates } \\
\sqsubseteq \forall \text { relatedObjects. } \\
\text { XtdObject } \\
\text { XtdRelAssociates } \\
\sqsubseteq \geq \mathrm{n} \text { relatedOb- } \\
\text { jects. } T \\
\text { XtdRelAssociates } \\
\sqsubseteq \leq \mathrm{n} \text { relatedOb- } \\
\text { jects. } T\end{array}$ & $\begin{array}{l}\text { <owl:ObjectProperty rdf:ID="relatingObject"> } \\
\text { < rdfs:subPropertyOf rdf:resource="\#relationship" } \\
> \\
\text { </owl:ObjectProperty> } \\
\text { <owl:ObjectProperty rdf:ID="relatedObjects"> } \\
\text { <rdfs:subPropertyOf rdf:resource="\#relationship" } \\
> \\
\text { </owl:ObjectProperty> } \\
\text { <owl:ObjectProperty rdf:ID="invRelationship"> } \\
\text { <owl:inverseOf rdf:resource="\#relationship"/> } \\
\text { </owl:ObjectProperty> } \\
\text { <owl:ObjectProperty } \\
\text { rdf:ID="invRelatedObjects"> } \\
\text { <owl:inverseOf rdf:resource="\#relatedObjects"/> } \\
\text { </owl:ObjectProperty> } \\
\text { <owl:ObjectProperty } \\
\text { rdf:ID="invRelatingObject"> } \\
\text { <owl:inverseOf rdf:resource="\#relatingObject"/> } \\
\text { </owl:ObjectProperty> } \\
\text { </owl:Class> }\end{array}$ \\
\hline
\end{tabular}

\subsection{Detection of Similarities}

The Detector of Similarities uses the ONDIL inference engine to detect similarities between each of two concepts belonging to different meta-schemas. The similarity between two concepts is defined in four levels according to its granularity.

Consider two concepts $C 1$ and $C 2$ belonging to different meta-schemas. Firstly, the inference engine verifies whether they are equivalent according to OWL semantics. If so, the result is sent to the Validator. If not, they are sent to the Subsumption Detection component to check if one concept is subsumed by (i.e. part of) the other. If that is not the case, similarity is evaluated by the Intersection Detection and LCS and 
Difference Detection components that take care of intersections, unions and differences among the concepts. This allows more precise detection of similarities between the two concepts. The similarities among the meta-schemas are validated in order to produce the OSIECS meta-schema.

\subsection{Matching the Entities}

The similarities found in the previous stage are used over the SRs schemas following a specialisation approach. Therefore, the Entities Matcher applies a similarity detected between two entities (at the meta-schema level) to the entities at the schema level. For instance, if $A$ is an entity from the e-COGNOS meta-schema and $B$ an entity from the ISO $12006-3$ meta-schema, then $S(A, B)$ represents a similarity between those entities. This similarity is matched to the entities of the corresponding eCOGNOS and LexiCon schemas, $S^{\prime}(a, b)$. All entities matched in the schema of the selected SRs comprise the OSIECS schema.

\section{Conclusions and Future Work}

The FUNSIEC framework supporting the interoperability of SRs for the construction sector has been presented with emphasis on the development of OSIECS infrastructure. The heart of such infrastructure is the OSIECS Kernel, which relies strongly on the OSIECS meta-schema/schema, both developed from the respective metaschemas/schemas of the SRs used in OSIECS. The OSIECS Kernel and the OSIECS Meta-schema/schema, to be released by the end of 2004, are the major results of the FUNSIEC project.

Both OSIECS meta-schema and schema are created semi-automatically by experts in a process aided by specially developed software tools. The FUNSIEC approach began with the analysis of the meta-schemas/schemas of the SRs chosen to form OSIECS. The selected SRs, represented in EXPRESS and UML, were converted to OWL, the neutral representation language adopted in FUNSIEC. For that, rules of conversion from EXPRESS/UML were manually generated to feed the JavaCC tool, which in turn generated two compilers to automatically transform any SRs from EXPRESS/UML to OWL. The converted meta-schemas were semantically compared and mapped. The final output is the OSIECS meta-schema, which is used afterwards to produce the respective OSIECS schema. The OSIECS infrastructure, now being developed, aims to prove the usefulness of these components.

The future of FUNSIEC remains full of challenges. The project is in its crucial phase where the concepts and ideas have to be demonstrated and assessed properly. Most of the OSIECS infrastructure will be in operation by January 2005 and by that time the underlying assumptions behind FUNSIEC should be confirmed. Specifically, the work started by translating EXPRESS expressions used in ISO-12006-3 into OWL (which produced the EXPRESS-OWL transformer). The extension of this process targets the translation of EXPRESS expressions used in IFC into OWL. Additionally, in order to guarantee the formal basis of the conversion its technical and 
semantical soundness and completeness are required to be studied. Finally, the inclusion of fuzzy logics to provide a degree of equivalence when mapping the metaschemas is another challenge facing FUNSIEC.

\section{References}

1. Lima, C., Fiès, B., Ferreira-da-Silva, C.: Setting up the Open Semantic Infrastructure for the Construction Sector in Europe - the FUNSIEC Project. In: 5th European Conference on Product and Process Modelling in the Building and Construction Industry - ECPPM 2004, Istambul, Turkey (2004)

2. Euzenat J., Le Bach T., Barrasa J., Bouquet P., De Bo, J., Dieng R. et al.: D2.2.3: State of the art on ontology alignment - Knowledge Web project, realizing the semantic web, IST2004-507482 Programme of the Commission of the European Communities (2004)

3. Benerecetti M., Bouquet P., Zanobini S.: Soundness of Semantic Methods for Schema Matching. Workshop on Meaning Coordination and Negotiation at the 3rd International Semantic Web Conference, Japan (2004)

4. Lima, C. P., Stephens, J., Bohms, M.: The bcXML - Supporting eCommerce and Knowledge Management in the construction industry. Itcon Journal, v. 8, (2003) p. 293-308

5. Lima, C. P., Fiès, B., Lefrancois, G., Diraby, T. E. (2003). The challenge of using a domain Ontology in KM solutions: the e-COGNOS experience. In: 10TH ISPE 2003, Funchal, Portugal. International Conference on Concurrent Engineering: Research and Applications, (2003) p. 771-778

6. The International Alliance for Interoperability web site, http://www.iaiinternational.org/iai_international/

7. ISO/DIS 12006-3 - Building construction Organization of information about construction works - Part 3: Framework for object-oriented information exchange (2002). http://www.icis.org/tc59sc13wg6/WG6-104\%20ISO_PAS_12006_3_VERSION_2X.pdf

8. European eConstruction Meta-Schema (EeM), CEN Workshop Agreement - CWA3. Documents produced by the CEN/ISSS eConstruction Workshop, Brussels (2004)

9. Le Duc, C.: Transformation d'ontologies basées sur la Logique de Description - Application dans le Commerce Electronique. PhD Thesis, Université de Nice, France (2004)

10. JavaCC, https://javacc.dev.java.net/

11. UML: Unified Modeling Language, Object Management Group, http://www.uml.org/

12. EXPRESS-G - annex A of ISO 10303-11 - Industrial automation systems and integration -Product data representation and exchange -- Part 11: Description methods: The EXPRESS language reference manual (1994)

13. Wix, J., Liebich, T. (2003) IFC: Current Reality and Future Potential, In Proceedings of $10^{\text {th }}$ ISPE International Conference on Concurrent Engineering: Research and Applications, Madeira, Portugal, July 2003.

14. CWA3 - CEN Workshop Agreement, European eConstruction Meta-Schema (EeM), 2004. Documents produced by the CEN/ISSS eConstruction Workshop, Brussels, 2004. 